\title{
Silent Patent Ductus Arteriosus Aneurysm
}

\author{
Aline Medeiros Botta, Flávio Aquino, Carla Pereira, Alberto Fin, Aldemir Nogueira, \\ Cláudia Ricachinewsky, Fernando Lucchese, Silvana Molossi
}

\author{
Porto Alegre, RS - Brazil
}

\begin{abstract}
Ductus arteriosus aneurysm, a rare and potentially fatal condition, has been reported as a complication after surgical ductus arteriosus closure. Its spontaneous appearance as a septic complication, which was common in the presurgical and preantibiotic era, has been rarely reported in the contemporary literature. Persistence of silent ductus arteriosus in healthy children and adults is a frequent condition that currently has an increasing diagnostic possibility due to the availability of more accurate investigative methods, especially echocardiography.

We report the case of a 1-year-old child, in whom no previous heart disease was known, who developed a giant aneurysm of the ductus arteriosus during a staphylococcal infection. This complication appeared after craniotomy for emptying an accidental subdural hematoma. This report associates the persistence of ductus arteriosus with a complication considered rare, which has a rapidly fatal evolution.
\end{abstract}

Patent ductus arteriosus aneurysm is a rare, but potentially fatal, condition reported both in children and adults ${ }^{1}$. It may have a spontaneous origin, including in fetuses, or an acquired origin as a complication after surgical closure of a persistent ductus arteriosus ${ }^{2}$.

Signs and symptoms are usually scarce and discrete. Its diagnosis is currently facilitated due to the availability of modern and accurate investigative methods of imaging, especially echocardiography ${ }^{3}$.

Patent ductus arteriosus in children and adults with no corresponding clinical manifestation has been progressively more evident on Doppler color echocardiography. This entity has been called the silent persistency of ductus arteriosus, whose natural history and management remain controversial.

Irmandade da Santa Casa de Misericórdia de Porto Alegre and Hospital de Clínicas de Porto Alegre - UFRGS

Mailing address: Aline Medeiros Botta - R. Desembargador Moreno Loureiro Lima, 85/801 - Bela Vista - 90450-130 - Porto Alegre, RS, Brazil - abotta@terra.com.br English version by Stela Maris C. e Gandour
The objective of this study was to report a case of aneurysmal dilation of a patent ductus arteriosus causing severe acute heart decompensation in a patient with no previous diagnosis of heart disease.

\section{Case report}

The patient is a 1-year-old male child who had been previously diagnosed with congenital multiplex arthrogryposis. The cardiological physical examination had been described as normal by the pediatrician. At that age, the patient underwent cranial trauma due to a fall to the ground, when craniotomy was required for draining a subdural hematoma. The patient had an infectious complication of the operative wound, in which Staphylococcus aureus was identified. The patient was treated with an intravenous antibiotic (ceftriaxone for 10 days) in his home town and was transferred to a tertiary hospital for neurosurgical follow-up. In that hospital, coagulase-negative St. aureus was identified in the blood culture, and the operative wound showed adequate scaring. The patient was treated with vancomycin for 7 days despite the absence of clinical signs of infection, being then discharged from the hospital. Two days after discharge, the patient returned to the emergency department with a lack of appetite, excessive crying, and tachycardia. On physical examination, the patient was in regular general condition, hydrated, slightly pale, and cried when touched. Cardiovascular examination revealed a hyperdynamic precordium with left ventricular impulsion, palpable thrill alongside the left sternal margin, systolic murmur (5+/6) audible in the entire precordium, tachycardia, and collapsing pulses. The results of laboratory tests were as follows: leukocytosis with a significant shift to the left and normal cerebrospinal fluid. The cerebral tomography was normal. The electrocardiography showed sinus tachycardia and biventricular hypertrophy. The chest X-ray showed a normal cardiac silhouette and pulmonary circulation, and widening of the superior mediastinum (fig. 1). The echocardiography revealed the following: situs solitus, levocardia, arch of the aorta to the left, extrinsic compression of the 
arch of the aorta due to severe dilation of the ductus arteriosus (aneurysm) measuring 3.0x $2.5 \mathrm{~cm}$; the flow in the descending a orta was slightly accelerated, with no significant gradient; the entrance orifice of the ductus arteriosus (aortic) measured $0.7 \mathrm{~cm}$ with a slightly restrictive flow; the exit orifice with moderate slightly restrictive flow to the pulmonary artery; slight enlargement of the left chambers (left atrium: $1.6 \mathrm{~cm}$, left ventricle: $2.9 \mathrm{~cm}$ ); a left ventricular hyperdynamic pattern. The remaining aspects of the cardiac anatomy were normal, and biventricular function was good (fig. 2). Considering the findings, emergency surgical intervention was indicated. In the operating room, the aneurysm ruptured in the perfusional entrance, which was immediately controlled, and aneurysmectomy was successfully completed. The patient received vancomycin for 21 days during the postoperative period, and was discharged from the hospital in good clinical condition with no sequelae. All posterior blood cultures were negative.

\section{Discussion}

Persistence of ductus arteriosus accounts for $5 \%$ to $10 \%$ of all congenital heart diseases, excluding premature

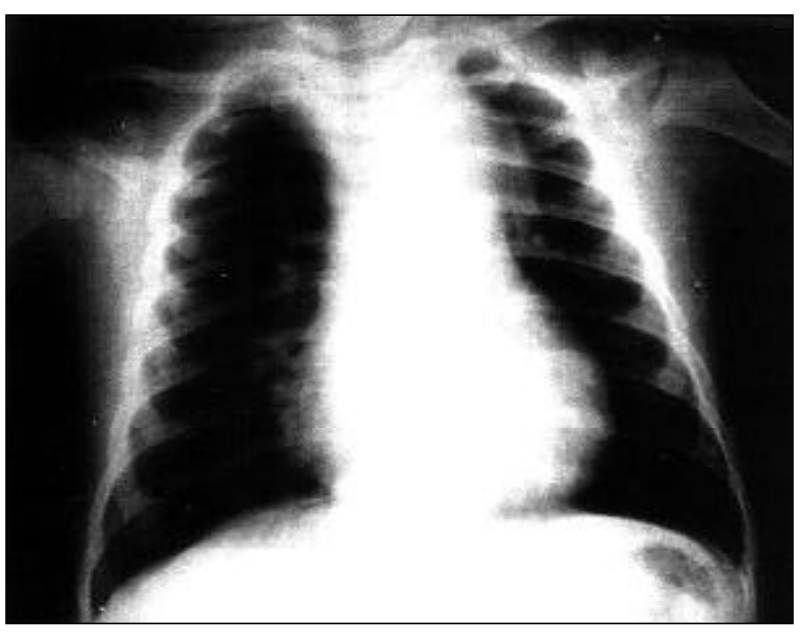

Fig. 1 - Chest X-rays.

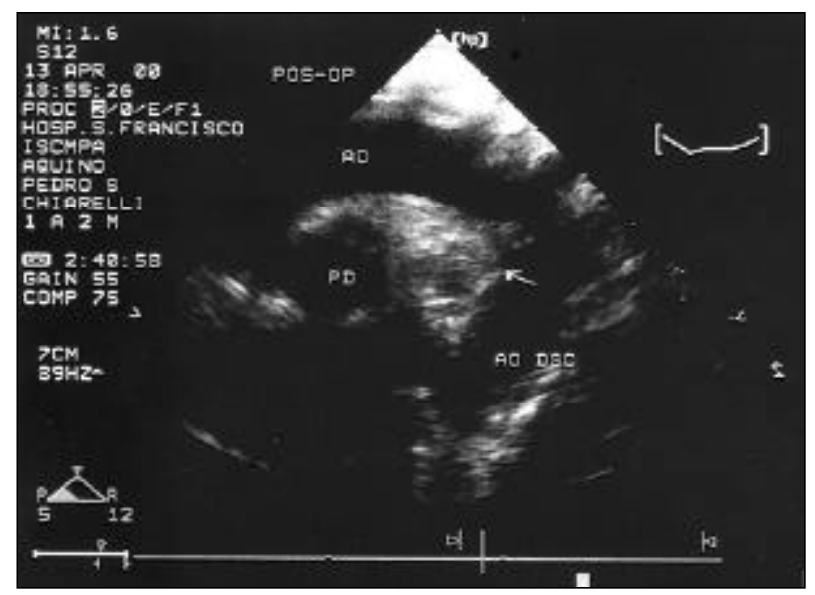

Fig. 2 - Echocardiography. newborns. This disease causes left-to-right shunt with increased pulmonary blood flow and left ventricular volume overload. The severity of the manifestations depends on the diameter of the ductus arteriosus. A small ductus arteriosus is usually well tolerated, but a large ductus arteriosus may lead to congestive heart failure and development of irreversible pulmonary vascular disease ${ }^{4}$. Heart auscultation is characterized by a machinery murmur of variable intensity depending on the diameter of the ductus arteriosus and degree of pulmonary resistance.

With the appearance of more sensitive diagnostic techniques, such as color Doppler echocardiography, small, clinically unidentifiable patent ductus arteriosus have been diagnosed in up to $0.5 \%$ of the children undergoing echocardiography for investigation of an innocent murmur ${ }^{3}$. Other authors report a $1 \%$ incidence of clinically silent persistent ductus arteriosus in children undergoing examinations due to Kawasaki's disease ${ }^{5}$.

Some series of autopsies published between 1936 and 1943, in the presurgical and preantibiotic era, showed high mortality in childhood due to persistence of ductus arteriosus. Infective endarteritis of ductus arteriosus was the most common cause of death accounting for $45 \%$ of the cases ${ }^{4,6}$. Currently, this risk is estimated to be much lower ${ }^{5}$. Complications of endarteritis in ductus arteriosus include septic embolism, mycotic aneurysm, and death ${ }^{7}$.

The incidence of an aneurysm of the ductus arteriosus is not known. A review published in 1991 found 144 case reports in the literature since 1827 . Prior to 1969 , the diagnosis was established on autopsy or on findings during exploratory thoracotomy. Since then, several cases have been diagnosed through noninvasive methods ${ }^{1}$.

The pathogenesis of ductus arteriosus also remains uncertain. In 1947, Helen Taussig explained that the delayed closure of the aortic side of the ductus arteriosus, exposing it to systemic pressures, was the cause of the aneurysmal formation. Other authors believe that this weakening may be congenital or acquired, such as consequent to an episode of infective endarteritis ${ }^{1,8}$. Connective tissue disorders (Marfan's disease) have been implicated and 4 cases have been reported ${ }^{1}$. No data exist in the literature about the relation between arthrogryposis multiplex congenita (disease of unknown cause characterized by contractures of joints and muscular hypodevelopment) and an increased risk of aneurysmatic dilation of ductus arteriosus. The cases reported about the formation of aneurysms after surgical treatment of persistent ductus arteriosus have been associated with the reestablishment of shunt and infectious complications (sepsis by Staphylococcus aureus) ${ }^{1,9}$.

Rupture, erosion to adjacent organs (bronchi, esophagus), infection, and thromboembolic events are the major complications of aneurysm. Rupture is the most frequently reported complication, occurring in $44 \%$ of the cases in children and in $28 \%$ of the cases in adults ${ }^{1,8}$.

Detection of an aneurysm of the ductus arteriosus is a mandatory indication for surgical treatment at any age ${ }^{1}$. 
Some cases identified in utero have been reported, which were followed with echocardiography, and were spontaneously closed by prenatal thrombosis ${ }^{10}$.

A consensus exists among the authors that most of the audible arterial ducts should be closed; the best approach for clinically silent persistent ductus arteriosus, however, is still controversial ${ }^{4}$. Some authors believe that the risk of infective endarteritis in silent persistent ductus arteriosus is still lower than that in patients with continuous murmurs. In their opinion, both from the clinical point of view and costeffectiveness, neither the elective surgical closure, nor the use of prophylaxis for bacterial endocarditis seem to be advantageous ${ }^{6,7,11-13}$. Other authors however report that even silent patent ductus arteriosus should be closed, because the risk of endarteritis cannot be overlooked. New techniques for ductus arteriosus closure through percutaneous devices have been used progressively more often with satisfactory results ${ }^{3,12,13}$.

In conclusion, the case reported is a confirmation that frightening complications of persistent ductus arteriosus, such as infective endarteritis and mycotic aneurysms, occur in cases of silent ductus arteriosus. Fortunetly, the immediate diagnosis and adequate surgical treatment have avoided disasters, such as rupture and possible death. However, doubts still persist about the advantage of the therapeutical intervention for all patients with silent persistent ductus arteriosus or even about the prophylaxis for bacterial endocarditis.

\section{References}

1. Lund JT, Jensen MB, Hjelms E. Aneurysm of the ductus arteriosus. A review of the literature and surgical implications. Eur J Cardiothorac Surg 1991; 5: 566-70.

2. Jesseph JM, Mahony L, Girod DA, et al. Ductus arteriosus aneurysm in infancy. Ann Thorac Surg 1985; 40: 620-2.

3. Salazar J, Oliván P, Ibarra F, et al. Ductus arteriosus permeable silente no complicado en niños. Diagnóstico por eco-Doppler. Rev Esp Cardiol 1990; 43: 410-2.

4. Radtke WAK. Current therapy of the patent ductus arteriosus. Curr Opin Cardiol 1998; 13: 59-65.

5. Thilén U, Aström-Olsson K. Does the risk of infective endarteritis justify routine patent ductus arteriosus closure? Eur Heart J 1997; 18: 503-6.

6. Sullivan IA. Patent arterial duct: when should it be closed? Arch Dis Child 1998; 78: 285-7

7. Huggon IC, Qureshi AS. Is the prevention of infective endarteritis a valid reason for closure of the patent arterial duct? Eur Heart J 1997; 18: 364-6.
8. Rangel-Abundis A, Baduí E, Verdin R, et al. Aneurisma espontaneo del conducto arterioso permeable, com endarteritis, presentacion de un caso. Arch Inst Cardiol Mex 1991; 61: 59-64.

9. Friedland IR, Plessis J, Cilliers A. Cardiac complications in children with Staphylococcus aureus bacteremia. J Pediatr 1995; 127: 746-8.

10. Maisel P, Brenner J. Spontaneous closure and thrombosis of a ductal aneurysm in a neonate. Cardiol Young 1999; 9: 503-5.

11. Lloyd TR, Beekman RH. Letter: Clinically silent patent ductus arteriosus. Am Heart J 1994; 127: 1664.

12. Rao PS. Transcatheter occlusion of patent ductus arteriosus: which method to use and which ductus to close. Am Heart J 1996; 132: 905-9.

13. Janorkar S, Goh T, Wilkinson J. Transcatheter closure of patent ductus arteriosus with the use of Rashkind occluders and/or Gianturco coils: long-term follow-up on 123 patients and special reference to comparison, residual shunt, complications and technique. Am Heart J 1999; 138: 1176-83. 